\title{
The evolution of runaway stellar collision products
}

\author{
E. Glebbeek ${ }^{1, \star}$, E. Gaburov ${ }^{2,3, \star \star}$, S. E. de Mink $^{1}$, O. R. Pols ${ }^{1}$, and S. F. Portegies Zwart ${ }^{2,3}$
}

\author{
1 Sterrekundig Instituut Utrecht, PO Box 80000, 3508 TA Utrecht, The Netherlands \\ 2 Sterrenkundig Instituut "Anton Pannekoek", Kruislaan 403, 1098 SJ Amsterdam, The Netherlands \\ 3 Section Computational Science, Kruislaan 403, 1098 SJ Amsterdam, The Netherlands
}

Received 19 June 2008 / Accepted 3 February 2009

\section{ABSTRACT}

\begin{abstract}
In the cores of young dense star clusters, repeated stellar collisions involving the same object can occur. It has been suggested that this leads to the formation of an intermediate-mass black hole. To verify this scenario we compute the detailed evolution of the merger remnant of three sequences, then follow the evolution until the onset of carbon burning, and estimate the final remnant mass to determine the ultimate fate of a runaway merger sequence.

We use a detailed stellar evolution code to follow the evolution of the collision product. At each collision we mix the two colliding stars, accounting for the mass loss during the collision. During the stellar evolution we apply mass-loss rates from the literature, as appropriate for the evolutionary stage of the merger remnant. We computed models for high $(Z=0.02)$ and low $(Z=0.001)$ metallicity to quantify metallicity effects.

We find that the merger remnant becomes a Wolf-Rayet star before the end of core hydrogen burning. Mass loss from stellar winds dominates the mass increase due to repeated mergers for all three merger sequences that we consider. In none of our high-metallicity models an intermediate-mass black hole is formed, instead our models have a mass of 10-14 $M_{\odot}$ at the onset of carbon burning. For low metallicity the final remnant is more massive and may explode as a pair-creation supernova. We find that our metal-rich models become inflated as a result of developing an extended low-density envelope. This may increase the probability of further collisions, but self-consistent $N$-body calculations with detailed evolution of runaway mergers are required to verify this.
\end{abstract}

Key words. stars: evolution - stars: formation - stars: mass-loss - galaxies: clusters: general

\section{Introduction}

The usual mode of star formation leads to a spectrum of masses between the theoretical hydrogen-burning limit and some upper limit, which appears to be close to about $100 M_{\odot}$ (Elmegreen 1999; Weidner \& Kroupa 2004; Figer 2005). In young and dense star clusters more massive stars can form when two or more high-mass stars coalesce. The cluster environment helps in driving these stars together. If this happens in a sufficiently young and dense star cluster the same star may experience multiple collisions in what is named a "collisional runaway" (Portegies Zwart et al. 1999). During such a chain collision several stars collide in short succession. The trigger for a chain collision is the gravothermal collapse (Bettwieser \& Sugimoto 1984) of the core of a young and dense star cluster. The density reached during core collapse depends on the cluster parameters. For example, for the cluster MGG-11 in the starburst galaxy M 82 ( $M=3.5 \times 10^{5} M_{\odot}, r=1.2$ pc McCrady et al. 2003), which was studied by Portegies Zwart et al. (2004), this leads to a central density $\rho_{\mathrm{c}} \simeq 10^{8} M_{\odot} / \mathrm{pc}^{3}$. If cluster core collapse is initiated before the most massive stars leave the main sequence (after about 3 Myr, Portegies Zwart et al. 2004; Gürkan et al. 2006)

\footnotetext{
* Current address: Department of Physics and Astronomy, McMaster University, 1280 Main Street West, Hamilton, Ontario, Canada L8S 4M1. e-mail: glebbeek@mcmaster.ca

$\star \star$ Current address: Leiden Observatory, PO Box 9513, 2300 RA Leiden, The Netherlands.
}

the collisional runaway sets in (Portegies Zwart \& McMillan 2002) and continues until the target star leaves the main sequence (Portegies Zwart et al. 1999).

If the star explodes as a supernova, this supernova is expected to be unusually bright and rich in hydrogen (Portegies Zwart \& van den Heuvel 2007). The star may also collapse completely into a black hole, without a visible supernova. The black hole remnant of such a star may be considerably more massive than hitherto observed (Portegies Zwart et al. 2004), though less massive than the supermassive black holes found in the nuclei of large galaxies (Kormendy \& Richstone 1995). Various groups have now confirmed the formation of such collisional runaways, and conjecture that the final merger product collapses to a black hole of up to about $10^{3} M_{\odot}$ (Portegies Zwart et al. 1999, 2004; Freitag et al. 2006; Gürkan et al. 2006). Multiple collisional runaways within the same simulation were found by Gürkan et al. (2006) for simulations with a binary fraction larger than 0.1 and these authors argue that multiple collisional runaways may be common in massive clusters.

Two of our aims in understanding merger runaways are understanding the structure of the merger remnant and the influence of stellar evolution of the merger runaway. Stellar evolution of very massive stars (with masses above $150 M_{\odot}$ ) has recently been studied by Belkus et al. (2007), Yungelson et al. (2008) and Langer et al. (2007). These studies all seem to come to the same conclusion: at high metallicity mass loss is copious enough to prevent the formation of a black hole of more than $50 M_{\odot}$, which is much lower than the conjectured intermediate-mass black hole mass. These studies, however, either used approximate formulae for stellar evolution (Belkus et al. 2007) or studied the evolution 
of very massive stars from the zero-age main sequence with an initially homogeneous composition (Yungelson et al. 2008; Langer et al. 2007). According to the dynamical simulations the massive star grows in mass by means of repeated collisions with less massive stars. The consequences of the collisions, the evolution between collisions and the differences in stellar age and structure at the moment of collision are not considered by these studies. A first attempt to overcome these problems by calculating collisions between massive stars and computing the evolution of these merged objects did not result in very different conclusions (Suzuki et al. 2007). Ideally, one would like to perform a fully self-consistent simulation in which the stellar dynamics, the hydrodynamics of the stellar collisions and the further evolution of the collision products are taken into account self consistently. Such multiscale simulations, however, will have to await the development of the appropriate numerical methodology. The MUSE software environment may provide the necessary functionality for such simulations ${ }^{1}$.

In this work we investigate the evolution of three collisional runaways that occurred in direct $N$-body simulations of the star cluster MGG-11 by Portegies Zwart et al. (2004). From their simulations we extract the masses of the stars involved in the collision sequences and the times of the collisions. We follow the evolution of the colliding stars with a stellar evolution code (described in Sect. 2.3) until the moment of collision. The outcome of the merger event is modelled as described in Sect. 2.2 and used as a new input model for the stellar evolution code. The evolution of the merged object is then followed until the next collision. Note that due to this approach the cluster dynamics is decoupled from the evolution of the collisional runaway. After the last collision we follow the evolution of the merger product until the evolution code fails to converge or until the onset of carbon burning, applying mass loss rates from the literature. We estimate the mass of the collision product at the end of the evolution and the mass of the black hole remnant. We also provide the chemical yields that result from the merger sequence and compare them to the combined yields of a population of normal single stars. Finally we comment on the effect of the initial composition, especially the heavy element content $Z$ (metallicity) on the structure of the merger remnant.

\section{Methods}

\subsection{Stellar dynamics}

The merger sequences used in this work were extracted from $N$-body simulations of young star clusters using the kira integrator of the starlab software environment (Portegies Zwart et al. 2001). These simulations used 131072 stars and ran on a special purpose GRAPE-6 computer (Makino 2001). In the original runs, stellar evolution was included using the prescription of Eggleton et al. (1989). Collisions were treated in a "sticky sphere" approximation: when the distance between two stars becomes less than the sum of their radii the stars are merged. The mass of the merged object is the sum of the masses of the colliding stars. The evolution of the merged star was continued using the same evolution prescription as used for normal single stars.

\subsection{Stellar collisions}

We use two different methods to model stellar collisions. The first assumes that the collision product is in hydrostatic and

\footnotetext{
${ }^{1}$ See http://muse.li.
}

thermal equilibrium and mixed homogeneously. The second method uses the prescription of Gaburov et al. (2008b) to model the structure of the remnant. In this case the collision product is not homogeneously mixed and it is not in thermal equilibrium (although it is still in hydrostatic equilibrium).

All collisions are treated as head-on collisions with vanishing velocity at infinity (i.e., parabolic collisions). We ignore rotation in this work despite the fact that rotation can have a significant influence on the evolution of a massive star (Maeder \& Meynet 2000b).

\section{Homogeneous mixing}

In this approach, detailed models of the progenitor stars were merged and homogeneously mixed at each step of the sequence. We assume that the merger remnant is in hydrostatic and thermal equilibrium. The mass loss from the collision is estimated according to Gaburov et al. (2008b).

In general merger remnants are not fully mixed (Lombardi et al. 1996; Gaburov et al. 2008b; Glebbeek et al. 2008; Gaburov et al. 2008a). However, Suzuki et al. (2007) find that their massive collision products are almost completely mixed during the subsequent evolution. Similarly, we find (Sect. 3.1) that the central convection zone in our merger remnants encompasses $\gtrsim 90 \%$ of the stellar mass. Rotational mixing, which we have ignored, will result in even more extended mixing of the collision product, so to first order homogeneous mixing is a reasonable approximation for our models.

\section{Detailed merger models}

Our detailed merger models were calculated using Make Me A Massive Star (MMAMS) (Gaburov et al. 2008b). The code has a prescription for the mass lost from the collision that is based on the results of smooth particle hydrodynamics calculations. Heating due to the dissipation of the kinetic energy of the progenitor stars in shocks and tides is also taken into account. After the ejected mass has been removed and heating has been applied to the material from the parent stars the structure of the collision remnant is determined by searching for a configuration that is dynamically stable (i.e. satisfies the Ledoux stability criterion, Kippenhahn \& Weigert 1990). An algorithm for doing this was first developed by Lombardi et al. (2002) for low-mass stars, for which it is sufficient to sort the mass shells in order of increasing entropy and then integrate the equation of hydrostatic equilibrium. For massive stars where radiation pressure is important this does not necessarily produce a stable configuration and some mass shells need to be moved again after the equation of hydrostatic equilibrium has been integrated. This procedure is repeated until a stable model is converged upon.

The output model is imported into the stellar evolution code using the method described in Glebbeek et al. (2008) and evolved until the time of the next collision.

Due to heating during the collision the merger product is not in thermal equilibrium. The excess of internal heat is radiated away during the contraction of the star to the main sequence. Because the stars were not in thermal equilibrium, we encountered more numerical problems when importing the stellar models than we did for the homogeneously mixed models. 


\subsection{Stellar evolution}

Our stellar evolution code is a version of the STARS code originally developed by Eggleton (1971) and later updated (e.g. Pols et al. 1995). This version of the code uses the opacities from Iglesias \& Rogers (1996) that take into account enhancement of $\mathrm{C}$ and O, as described in Eldridge \& Tout (2004) and Ferguson et al. (2005). The assumed heavy-element composition is scaled to solar abundances (Anders \& Grevesse 1989). Chemical mixing due to convection (Böhm-Vitense 1958; Eggleton 1972) is taken into account.

STARS uses an adaptive mesh in which the mesh points automatically redistribute themselves according to a mesh spacing function that places more meshpoints in regions of the star where a higher resolution is required. For the models presented here we used 200 mesh points for the main sequence phase and 500 for the core helium-burning evolution. Because our stars form an extended low-density envelope (see Sect. 3.1) we found it necessary to increase the number of mesh points in the outer layers compared to our standard stellar models.

We use a mass fraction of heavy elements $Z=0.02$ for our standard runs. In order to study the effect of metallicity we also recalculated one of our sequences with $Z=0.001$. Metallicity affects the mass-loss rate and therefore the mass of the progenitors at each collision. We terminate the evolution at central carbon ignition and estimate the final remnant mass according to the prescription of Belczynski et al. (2002).

\subsection{Mass loss}

Since our collision products become very massive and luminous, even exceeding the Humphreys-Davidson limit (a luminosity cutoff above which few stars are observed, Humphreys \& Davidson 1979), mass loss plays a key role in their evolution. Unfortunately, neither observations nor theoretical models of mass loss exists for the full range of masses and luminosities reached by our models. From the mass loss rates available in the literature we have adopted the theoretical rate from Vink et al. (2000, 2001, hereafter referred to as the Vink rate) in the temperature range $10000 \mathrm{~K} \lesssim T_{\text {eff }} \lesssim 50000 \mathrm{~K}$. For lower effective temperatures we use the empirical rate from de Jager et al. (1988, hereafter referred to as the de Jager rate).

All our models become helium-rich and evolve into WolfRayet (WR) stars. We follow the criterion used by Eldridge \& Vink (2006) to decide when our stars become WR stars. Specifically, we apply the mass-loss rate from Nugis \& Lamers (2000) when the surface abundance of hydrogen drops below 0.4 by mass fraction and $T_{\text {eff }}>10000 \mathrm{~K}$. In our models this happens before the star finishes core hydrogen burning (as in Langer et al. 2007; Yungelson et al. 2008). For our low metallicity run we used the metallicity scaling found by Vink \& de Koter (2005) for the WR mass-loss rate. The Vink et al. (2001) rate already includes metallicity scaling.

Apart from the de Jager and Vink rates, we also considered the mass loss rate from Kudritzki (2002). None of these rates fully cover the range of parameters of our models. The empirical de Jager rate has too few data points for luminosities above the Humphreys-Davidson limit. Their 20 point Chebyshev fit needs to be extended beyond $\log L / L_{\odot}=6.7$. The Vink rate is based on atmosphere models calculated for $\log L / L_{\odot}<6.25$ and $10000 \mathrm{~K} \lesssim T_{\text {eff }} \lesssim 50000 \mathrm{~K}$ and the Kudritzki rate needs to be extrapolated for $T_{\text {eff }}<40000 \mathrm{~K}$.

Our preference for the Vink rate comes from the fact that it best covers the range of effective temperatures for our models

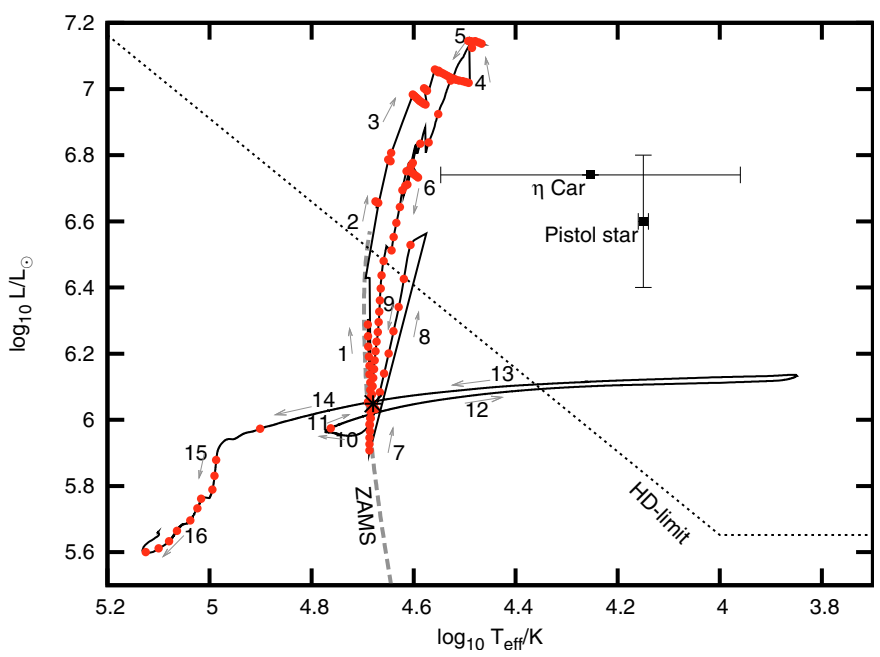

Fig. 1. Evolutionary track of the merger from sequence 1 in the Hertzsprung-Russell diagram. The starting point is indicated by $*$ and numbered arrows indicate the way the star evolves along the evolution track. Points are plotted on the evolutionary track after each $30000 \mathrm{yr}$. The dotted line indicates the Humphreys-Davidson limit. For reference, the theoretical ZAMS (running up to $200 M_{\odot}$ ) and the locations of $\eta$ Carinae and the Pistol Star are also plotted.

and can be better extrapolated in luminosity than the de Jager rate. By contrast, the Kudritzki rate needs to be extrapolated to lower effective temperatures, which is less reliable than extrapolating to higher luminosities because the presence of spectral lines that drive the wind is sensitive to the temperature. In the region where the Kudritzki rate is valid it is very similar to our extrapolated Vink rate. The Vink rate is not applicable to red supergiants and predicts a mass loss rate that is too low for cool stars. For this reason we adopt the de Jager rate rather than the Vink rate at effective temperatures below $10000 \mathrm{~K}$. Our models only reach this temperature for luminosities that are within the validity range of the de Jager rate.

Our adopted mass-loss rate likely underestimates the true mass loss rate since our stars are much closer to their Eddington limit than the model calculations on which the Vink rate is based. We will return to this point in the discussion.

\subsection{Rotation}

It has been shown that for off-axis collisions the angular momentum of the collision product can be so large that it cannot reach thermal equilibrium before losing a large fraction of its angular momentum (Lombardi et al. 1996; Sills et al. 1997). We may therefore underestimate mass loss from the collision. The mechanism for this angular momentum loss is unclear but it has been suggested that magnetic fields can play a key role (Leonard \& Livio 1995; Sills et al. 2005). Rapid rotation can also enhance the mass-loss rate of a star, especially close to the Eddington limit (Maeder \& Meynet 2000a). This increases the uncertainty in the mass-loss rate.

Rotation also influences the star through various instabilities that can induce mixing (Endal \& Sofia 1976; Pinsonneault et al. 1989; Heger et al. 2000). This mixing is important because it can bring helium to the surface, affecting the opacity of the envelope and increasing the luminosity and effective temperature of the star. As mentioned above, rotational mixing is not expected to alter the outcome of our calculations very strongly because our collision products are almost fully convective. 
Table 1. Parameters and results of the first collision sequence studied in this paper. Time $t$ in Myr, masses are in solar units. The final row gives the age at which our evolution calculations stopped and the mass of the collision product at the end of the evolution (assumed black hole mass).

\begin{tabular}{rrrrrrr}
\hline \hline$N$ & $t$ & $M_{1, N \text {-body }}^{a}$ & $M_{1, \text { mix }}^{a}$ & $M_{1, \mathrm{MMS}}^{a}$ & $M_{2}^{a}$ & $M_{\text {merger }}^{a}$ \\
\hline 1 & 0.22577 & 92.4 & 90.9 & 90.9 & 79.4 & 154.3 \\
2 & 0.22782 & 171.8 & 154.3 & 152.8 & 85.3 & 217.1 \\
3 & 0.23702 & 257.1 & 216.7 & 213.6 & 7.7 & 223.3 \\
4 & 0.31217 & 264.8 & 219.8 & 215.3 & 77.8 & 274.4 \\
5 & 0.40122 & 342.6 & 268.2 & 261.6 & 13.8 & 279.7 \\
6 & 0.43480 & 356.4 & 277.2 & 268.0 & 71.9 & 327.1 \\
7 & 0.43480 & 428.3 & 327.1 & 315.1 & 79.4 & 382.2 \\
8 & 0.74123 & 507.7 & 348.2 & & 30.1 & 371.8 \\
9 & 0.83549 & 537.7 & 360.2 & & 66.7 & 406.7 \\
10 & 0.90575 & 604.5 & 397.3 & & 1.2 & 398.5 \\
11 & 1.29919 & 605.7 & 353.5 & & 98.2 & 415.2 \\
12 & 1.33450 & 703.9 & 411.0 & & 24.2 & 430.8 \\
13 & 1.42468 & 728.0 & 417.6 & & 8.9 & 425.7 \\
14 & 1.50261 & 736.9 & 409.0 & & 2.2 & 411.2 \\
15 & 1.60190 & 739.1 & 216.7 & & 60.2 & 256.5 \\
16 & 1.63186 & 799.4 & 221.0 & & 9.2 & 228.9 \\
17 & 1.68112 & 808.5 & 186.0 & & 81.1 & 237.1 \\
18 & 1.75684 & 889.6 & 177.3 & & 40.9 & 205.5 \\
19 & 1.90688 & 930.5 & 193.6 & & 31.6 & 216.3 \\
20 & 2.07245 & 961.9 & 129.9 & & 2.6 & 132.2 \\
21 & 2.59778 & 963.1 & 47.6 & & 55.2 & 88.6 \\
22 & 3.10786 & 1012.7 & 40.9 & & 75.4 & 110.1 \\
& 3.72835 & 1118.9 & 13.9 & & & \\
\hline
\end{tabular}

a The primary mass according to the $N$-body code $M_{1, N \text {-body }}$ and according to our fully mixed models $M_{1, \text { mix }}$, the mass of the secondary $M_{2}$ and the mass of the fully mixed remnant after the collision $M_{\text {merger }}$. The mass $M_{1, \mathrm{MMS}}$ according to the MMAMS models we were able to calculate is given for comparison.

${ }^{b}$ See text.

Table 2. As Table 1 for collision sequence 2.

\begin{tabular}{rrrrrr}
\hline \hline$N$ & $t$ & $M_{1, N \text {-body }}$ & $M_{1, \text { mix }}$ & $M_{2}$ & $M_{\text {merger }}$ \\
\hline 1 & 0.72251 & 91.8 & 86.5 & 81.9 & 150.1 \\
2 & 0.72252 & 173.7 & 150.1 & 80.2 & 206.7 \\
3 & 0.88257 & 253.8 & 198.9 & 68.6 & 245.6 \\
4 & 0.88522 & 322.5 & 245.4 & 72.9 & 294.5 \\
5 & 0.95998 & 395.4 & 287.7 & 1.8 & 289.4 \\
6 & 1.41050 & 397.1 & 250.0 & 95.8 & 309.6 \\
7 & 1.50639 & 492.9 & 298.4 & 86.1 & 352.3 \\
8 & 1.97200 & 578.8 & 282.1 & 97.6 & 338.2 \\
9 & 2.22324 & 676.2 & 79.8 & 75.2 & 130.4 \\
10 & 2.22325 & 751.5 & 130.2 & 55.4 & 166.3 \\
11 & 2.45320 & 806.7 & 83.9 & 23.5 & 100.4 \\
12 & 2.68305 & 829.8 & 66.7 & 28.4 & 86.8 \\
13 & 2.74781 & 858.0 & 78.8 & 1.0 & 79.8 \\
14 & 3.08766 & 857.3 & 53.5 & 82.6 & 105.2 \\
15 & 3.19983 & 939.2 & 46.1 & 56.3 & 86.3 \\
16 & 3.27682 & 995.0 & 64.8 & 41.6 & 93.6 \\
& 4.51074 & 1036.6 & 10.1 & & \\
\hline
\end{tabular}

\section{Results}

The initial conditions and outcome of each of our merger sequences are listed in Tables 1-3. The tables give the time of collision $t$ as well as the masses $M_{1}$ and $M_{2}$ of the colliding stars and the mass $M_{\text {merger }}$ of the merged object. In the following we will refer to the object undergoing the subsequent merger events as the primary and to the star colliding with it as the secondary. Note that the mass of the primary depends on the treatment of
Table 3. As Table 1 for collision sequence 3.

\begin{tabular}{rrrrrr}
\hline \hline$N$ & $t$ & $M_{1, N \text {-body }}$ & $M_{1, \text { mix }}$ & $M_{2}$ & $M_{\text {merger }}$ \\
\hline 1 & 0.53120 & 77.8 & 75.3 & 70.7 & 131.5 \\
2 & 0.57294 & 148.5 & 130.7 & 82.8 & 191.3 \\
3 & 0.65612 & 231.3 & 188.1 & 58.5 & 228.6 \\
4 & 0.89154 & 289.8 & 215.4 & 96.6 & 279.9 \\
5 & 0.89155 & 386.4 & 279.9 & 78.5 & 332.5 \\
6 & 1.02208 & 464.9 & 318.1 & 1.5 & 319.5 \\
7 & 1.31086 & 466.4 & 289.3 & 56.3 & 327.9 \\
8 & 1.50575 & 522.6 & 305.7 & 48.9 & 340.1 \\
9 & 2.05327 & 571.3 & 164.0 & 72.7 & 209.2 \\
10 & 2.27966 & 643.8 & 73.1 & 2.0 & 74.8 \\
11 & 2.60213 & 645.0 & 35.1 & 3.5 & 37.7 \\
12 & 3.12235 & 642.8 & 19.8 & 49.3 & 60.7 \\
& 4.59400 & 692.1 & 9.7 & & \\
\hline
\end{tabular}

Table 4. As Table 3 but for $Z=0.001^{a}$.

\begin{tabular}{rrrrrr}
\hline \hline$N$ & $t$ & $M_{1, N \text {-body }}$ & $M_{1, \operatorname{mix}}$ & $M_{2}$ & $M_{\text {merger }}$ \\
\hline 1 & 0.53120 & 77.8 & 77.7 & 70.7 & 135.4 \\
2 & 0.57294 & 148.5 & 135.4 & 82.8 & 198.3 \\
3 & 0.65612 & 231.3 & 198.1 & 58.5 & 239.6 \\
4 & 0.89154 & 289.8 & 239.1 & 96.6 & 308.5 \\
5 & 0.89155 & 386.4 & 308.5 & 78.5 & 364.2 \\
6 & 1.02208 & 464.9 & 363.5 & 1.5 & 364.9 \\
7 & 1.31086 & 466.4 & 363.4 & 56.3 & 404.7 \\
8 & 1.50575 & 522.6 & 403.4 & 48.9 & 440.2 \\
9 & 2.05327 & 571.3 & 434.9 & 72.7 & 487.5 \\
10 & 2.27966 & 643.8 & 390.1 & 2.0 & 392.0 \\
11 & 2.60213 & 645.0 & 260.1 & 3.5 & 263.3 \\
& 2.83153 & 648.5 & $171.9^{b}$ & & \\
\hline
\end{tabular}

a Note that the merger sequence was terminated earlier than for $Z=$ 0.02 and that the merger remnant did not finish its evolution before the evolution code broke down.

$b$ Mass and time at the moment the evolution code broke down, rather than the onset of carbon burning.

the collisions and is different for each run. Sequence three was recalculated for $Z=0.001$ and the results for this run are given in Table 4.

The evolutionary track in the Hertzsprung-Russell diagram (HRD) for the first merger sequence (Table 1) is shown in Fig. 1. The two other sequences are similar. The location of the ZAMS (up to $200 M_{\odot}$ ) is indicated with a dashed line and every $30000 \mathrm{yr}$ is marked with $\bullet$. The repeated collisions drive the collision product to high luminosities, exceeding the HumphreysDavidson limit, but the collision product never moves far from the extension of the ZAMS, instead evolving nearly vertically in the HR diagram (similar to the evolutionary tracks for homogeneously evolving stars, Yoon \& Langer 2005). For reference, the locations of the Pistol Star and $\eta$ Carinae are also shown. The location of $\eta$ Car is based on Hillier et al. (2001), with the errorbar in effective temperature due to the range in their radius estimates. The luminosity is based on the infrared flux. The location of the Pistol Star is based on the low luminosity solution of Figer et al. (1998). We see that the merger remnant is always hotter than either of these two stars, except when it becomes a red supergiant (the red loop in Fig. 1), at which time it is less luminous. During the merger sequence the luminosity can exceed the luminosity of these stars. On the other hand, $\eta$ Carinae and the Pistol Star are both obscured by optically thick outflows, which means that comparing with the effective temperature of our model can be misleading because we do not model such 


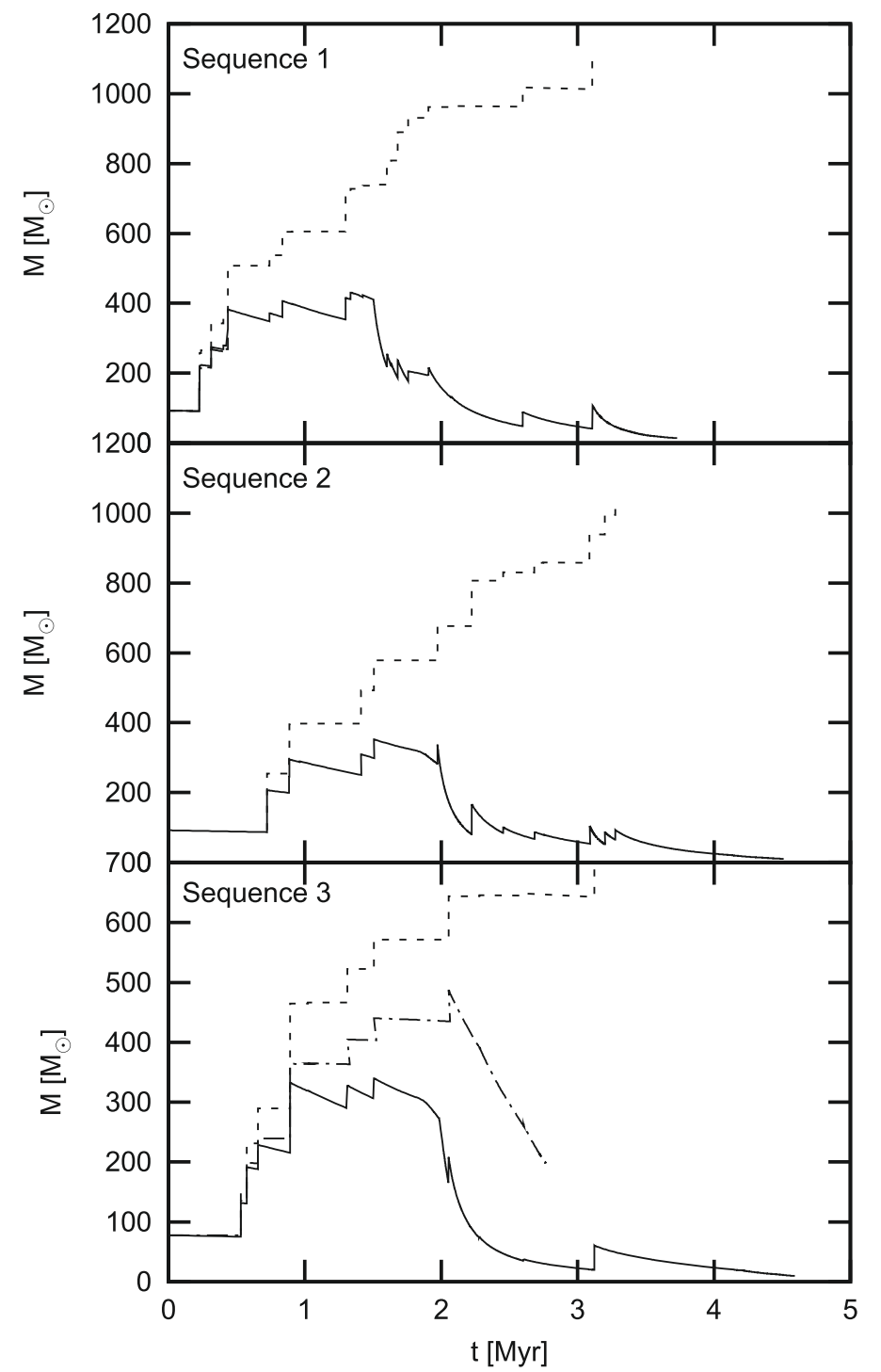

Fig. 2. The mass of the merger product as a function of time for the three merger sequences listed in Tables 1-3. The solid line is the mass found from the detailed models assuming homogeneous mixing, the dashed line is the mass predicted from the $N$-body calculation. The dash-dotted line in the bottom panel is the mass of the $Z=0.001$ run.

an optically thick wind. The location of the collision product above the Humphreys-Davidson limit suggests that it is a luminous blue variable (LBV) star, so that in reality its position in the HRD is likely to be variable.

The high luminosity increases the mass-loss rate, leading to a competition between mass loss due to stellar winds and mass increase due to collisions (see also Suzuki et al. 2007; Belkus et al. 2007). The time evolution of the mass of the mergers is shown in Fig. 2. The dashed lines give the mass that was predicted in the $N$-body simulation (assuming no mass is lost in the collision) while the solid lines show our fully mixed models for $Z=0.02$. The two agree well for the first few collisions, but mass loss due to stellar wind prevents the mass from exceeding $500 M_{\odot}$. The surface of the merger remnants becomes heliumrich and after 1.5-2 Myr turns the star into a Wolf-Rayet star. The strong WR mass-loss rate (up to $3.6 \times 10^{-3} M_{\odot} \mathrm{yr}^{-1}$ when the collision product first becomes a WR star) brings the mass down on a timescale of $10^{4}-10^{5} \mathrm{yr}$, to $\sim 100 M_{\odot}$ after the final collision and 10-14 $M_{\odot}$ at the time of carbon ignition.

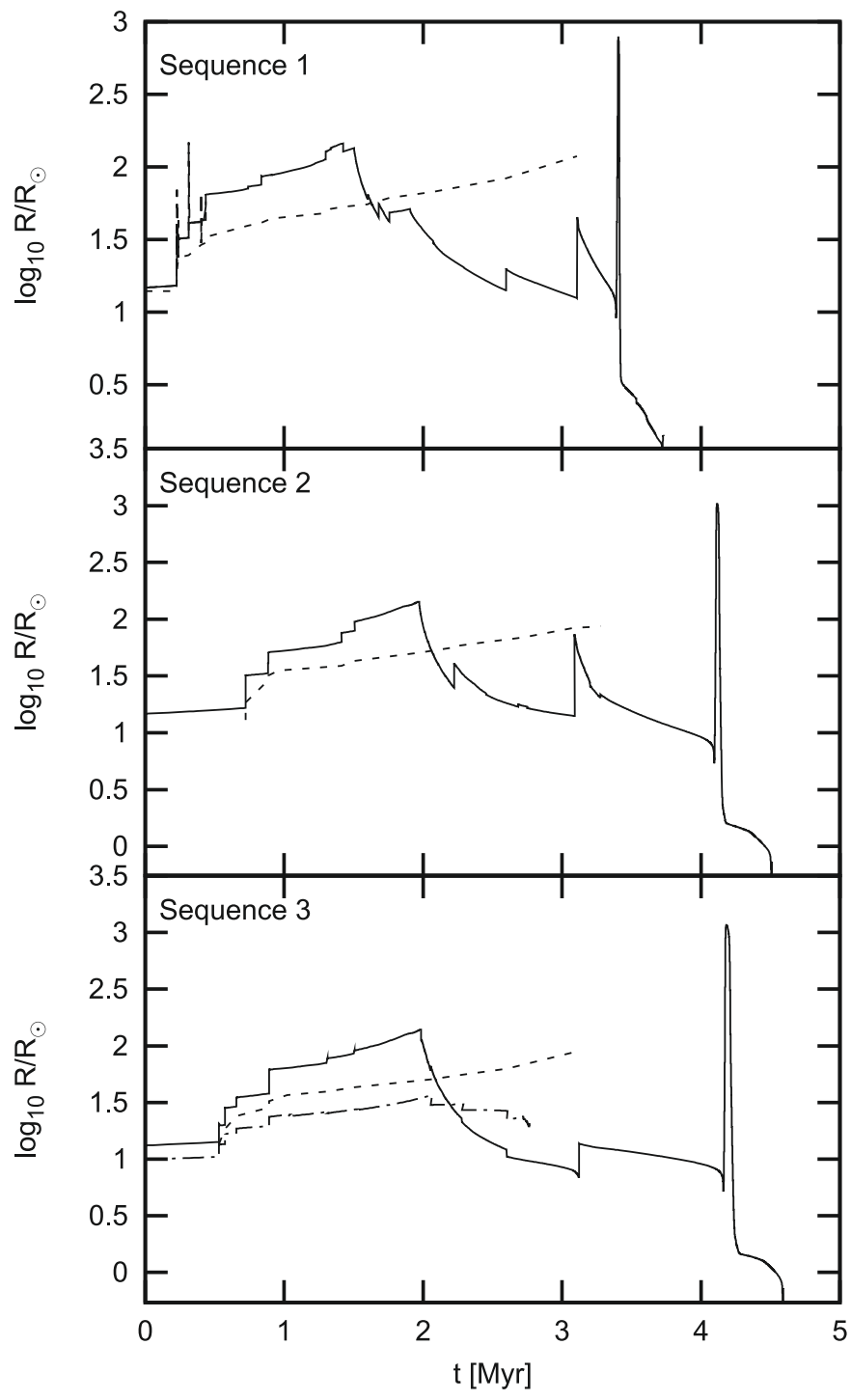

Fig. 3. The radius of the merger remnant of the three sequences against time. The solid line is the prediction from our homogeneous models while the dashed line is the radius from the $N$-body code. The long dashed line in the top panel is the radius from the MMAMS model, which shows spikes at each collision because the merger remnant is out of thermal equilibrium immediately after the merger. The dash-dotted line in the bottom panel is the radius of the $Z=0.001$ run.

In Fig. 3 we follow the evolution of the radius. Despite the lower mass our collision products have substantially larger radii (up to a factor three, note the logarithmic scale) than was assumed by the $N$-body code. This is due to a peculiarity in the structure of the collision product, which will be discussed in detail in Sect. 3.1. When the collision product becomes a WR star, the radius decreases substantially and the collision product can be up to an order of magnitude smaller than was assumed in the $N$-body calculation. After core hydrogen exhaustion the collision product still has a thin hydrogen-poor $(X \approx 0.04)$ envelope. Expansion of this envelope is responsible for turning the star into a red supergiant and causes the spike in the radius at $3.5 \mathrm{Myr}$ (first sequence) and 4.1 Myr (second and third sequence). During the red supergiant phase the collision product is again above the Humphreys-Davidson limit, but this phase is very short, lasting $17 \times 10^{3} \mathrm{yr}(<1 \%$ of the lifetime of the star $)$. 
Table 5. Times $t$ and secondary masses $m_{2}$ for the sub merger sequence leading to the secondary of collision 22 in the merger sequence in Table 1.

\begin{tabular}{rrrrrr}
\hline \hline$N$ & $t$ & $M_{1, N \text {-body }}$ & $M_{1, \text { mix }}$ & $M_{2}$ & $M_{\text {merger }}$ \\
\hline 1 & 0.88048 & 66.6 & 63.6 & 65.2 & 113.4 \\
2 & 1.08887 & 131.8 & 110.4 & 16.4 & 114.1 \\
& 3.10786 & 106.2 & 75.4 & & \\
\hline
\end{tabular}

We have also plotted the mass and radius from the MMAMS models that we were able to calculate. In the upper panel of Fig. 2 the MMAMS model is indistinguishable from the homogeneous model. After the collision the merger remnant is out of thermal equilibrium and is inflated. The increase in radius at each collision can be seen in the upper panel of Fig. 3. Once the collision product reaches thermal equilibrium (after $\sim 10^{4} \mathrm{yr}$ ) the radius closely follows the radius of the homogenised model, indicating that the homogenised model is indeed a reasonable approximation of the structure of the merger remnant. Because the radius is larger while the collision product is out of thermal equilibrium it is more likely to interact or collide with other stars at this time, but since we do not take feedback on the dynamics into account this effect is not important for our present considerations.

The collision sequences mostly involve main-sequence stars but a few of the listed collisions are special. Collision 22 of the first sequence involves the remnants of two collision runaways. The star that has undergone the longest sequence of collisions (the "primary") is an early type Wolf-Rayet star at this point with a surface hydrogen abundance of 0.24 and a mass of $41 M_{\odot}$. However, it is still undergoing core hydrogen burning. The star that has undergone the shortest merger sequence (the "secondary", see Table 5) is a core helium burning star of $75 M_{\odot}$ that has not yet become a Wolf-Rayet star although its surface hydrogen abundance is 0.44 , which is close to our criterion. In our homogeneous mixing treatment the result is a collision product that has been enhanced in carbon (see the surface abundance plot in Fig. 5), which is converted into nitrogen through $\mathrm{CNO}$ processing. In a more detailed treatment of the merger process we expect the dense helium core of the secondary to sink to the centre of the collision product so that the merger remnant would have a hydrogen depleted core.

A similar situation occurs for collision 14 from the second sequence, for which the secondary has also become a core helium-burning star at the time of collision.

\subsection{Structure and size of the merger remnants}

Apart from the mass the size of the merger remnant is one of the parameters that determines the probability of subsequent collisions in a cluster. As mentioned, the large radius of the $Z=0.02$ models during the first 1.5-2 Myr in Fig. 3 is caused by a peculiarity of the stellar structure.

The merger remnants become very massive and are almost fully convective. The lower panel in Fig. 4 shows the location of convection zones against the enclosed mass, the upper panel shows the same information as a function of the radius. The central convection zone contains about $90 \%$ by mass of the merger remnant. As can be seen from the figure, mass loss between the collisions can reveal material from the central convection zone at the stellar surface (for instance, the mass of the star at $1.3 \mathrm{Myr}$ is lower than the mass of the convective core at $0.8 \mathrm{Myr}$ ).

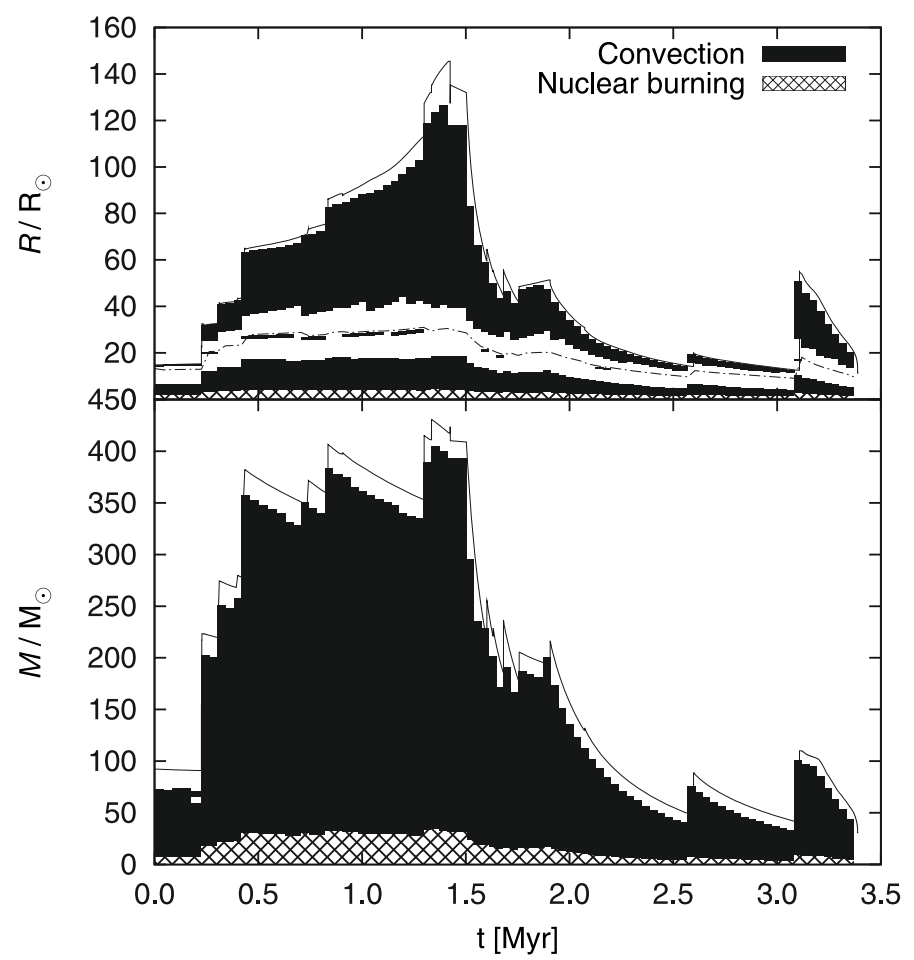

Fig. 4. Kippenhahn diagram showing the evolution of the collision product of the first merger sequence. The plot shows convection zones against the radius (upper panel) and against mass coordinate (lower panel) as a function of time. In the upper panel the radius containing a fraction 0.99999 of the stellar mass is indicated with a dash-dotted line. The convective core encompasses $90 \%$ of the mass but a much smaller fraction of the radius (at most $20 R_{\odot}$ ). Mass loss between collisions exposes material from the convective core.

The outer $10 \%$ by mass of the merger remnant is radiative, but the upper panel of Fig. 4 shows two convection zones in this region which correspond to different peaks in the opacity of the stellar material. An extended convective layer that corresponds to the "Fe bump" is located at large radii. The Fe bump is an increase in opacity around $\log T \approx 5.3$ which was found after introducing the treatment of spin-orbit splitting of iron and nickel into the computation of the opacity tables (Rogers \& Iglesias 1992; Seaton et al. 1994). Deeper down a thin convective layer can be seen, caused by the "deep Fe bump", occurring around $\log T \approx 6.3$.

Together, these convection zones are very extended in radius but contain almost no mass. It is especially the convection zone corresponding to the Fe-bump which expands even more while the star evolves. When the star reaches its maximum radius of about $150 R_{\odot}$ after $1.4 \mathrm{Myr}$, this convection zone extends over $90 R_{\odot}$, while it contains only about $10^{-4} M_{\odot}$. At this moment the star consists of a core of less than $30 R_{\odot}$ in size containing almost all of the mass surrounded by an extended "halo" reaching from 30 to $150 R_{\odot}$. This halo has an almost constant temperature and density of $10^{-10} \mathrm{~g} \mathrm{~cm}^{-3}$. This is indicated in Fig. 4, which shows the radius outside which a fraction $10^{-5}$ of the stellar mass is located.

This "core-halo" structure has been found before in models of massive stars, for example by Ishii et al. (1999) for hydrogenrich stars and by Petrovic et al. (2006) for helium stars. Petrovic et al. (2006) note that to provide the high mass-loss rate from the surface a high outward velocity is needed in the outer layers where the density is low. In their models the necessary velocity 
Table 6. Ejected mass and composition for the three computed merger sequences compared to the winds of a population of single stars corresponding to sequence 1 .

\begin{tabular}{|c|c|c|c|c|c|c|c|c|c|c|c|c|c|}
\hline & Single & \multicolumn{4}{|c|}{ Sequence 1} & \multicolumn{4}{|c|}{ Sequence 2} & \multicolumn{4}{|c|}{ Sequence 3} \\
\hline & Wind & Coll. & Wind & Remnant & Total & Coll. & Wind & Remnant & Total & Coll. & Wind & Remnant & Total \\
\hline$\Delta M$ & 647.5 & 228.6 & 695.8 & 95.4 & 1020 & 191.8 & 549.9 & 83.5 & 825 & 142.3 & 456.6 & 102.0 & 701 \\
\hline $\mathrm{H}$ & 0.4806 & 0.5648 & 0.4006 & 0.0899 & 0.4083 & 0.5599 & 0.3489 & 0.1944 & 0.3823 & 0.5521 & 0.3361 & 0.2025 & 0.3605 \\
\hline $\mathrm{He}$ & 0.4965 & 0.4000 & 0.5794 & 0.8308 & 0.5627 & 0.4093 & 0.6311 & 0.7637 & 0.5930 & 0.4305 & 0.6439 & 0.7553 & 0.6168 \\
\hline $\mathrm{C}$ & 0.0040 & 0.0012 & 0.0003 & 0.0306 & 0.0033 & 0.0090 & 0.0003 & 0.0133 & 0.0036 & 0.0004 & 0.0003 & 0.0184 & 0.0030 \\
\hline $\mathrm{N}$ & 0.0092 & 0.0166 & 0.0132 & 0.0294 & 0.0155 & 0.0117 & 0.0133 & 0.0128 & 0.0129 & 0.0105 & 0.0134 & 0.0123 & 0.0127 \\
\hline $\mathrm{O}$ & 0.0040 & 0.0013 & 0.0010 & 0.0060 & 0.0015 & 0.0044 & 0.0009 & 0.0081 & 0.0024 & 0.0013 & 0.0008 & 0.0050 & 0.0015 \\
\hline
\end{tabular}

The first row lists the total mass $\Delta M$ (in solar units) lost through each of the three listed channels, the remaining rows give the abundances (by mass fraction) of $\mathrm{H}, \mathrm{He}, \mathrm{C}, \mathrm{N}$ and $\mathrm{O}$. For each sequence the first column lists the ejecta from the collision, the second column lists the integrated values for the stellar wind during the merger sequence, the third column lists the values for the evolution of the merger remnant after the merger sequence ended and the fourth column gives the combined yields from these channels.

is higher than the local sound speed by an order of magnitude. This means that the halo cannot be modelled realistically under the assumption of hydrostatic equilibrium and may not be stable. They find that with a more detailed treatment the halo disappears as a result of the high mass-loss rate. Because our merger remnants have a much larger radius the outflow velocity in the halo is about 2-3 orders of magnitude smaller than the sound speed, which suggests that the halo structure is stable in this case. The halo disappears when the merger remnants become Wolf-Rayet stars and the mass-loss rate increases.

\subsection{Final remnant masses}

For each of the three merger sequences the collision product is close to core hydrogen exhaustion when the merger sequence ends. After the end of the main sequence hydrogen continues to burn in a shell very close to the surface. The hydrogen envelope expands, driving the star into a red loop in the HRD. Mass loss from the surface gradually removes the hydrogen envelope, reducing the efficiency of the hydrogen burning shell. When the hydrogen shell is extinguished the star returns to the blue part of the HRD. The remaining hydrogen envelope is lost and the star becomes a massive $\left(\sim 20-30 M_{\odot}\right)$ helium star. By the end of core helium-burning the mass has gone down to $10-14 M_{\odot}, 80 \%$ of which is taken up by the $\mathrm{C} / \mathrm{O}$ core. The expected outcome of the evolution for such stars is a complete collapse to a black hole. In each of these sequences $600-900 M_{\odot}$ is lost to the interstellar medium (see Table 6).

\subsection{Surface abundances and chemical yields}

In the course of its evolution the surface of the merger remnant gradually becomes helium-rich, as can be seen in the top panel of Fig. 5. The CNO abundances (shown in the bottom panels of Fig. 5) change strongly at the first collision and then stay mostly constant up to $t \approx 3 \mathrm{Myr}$.

The abundances change most strongly during collisions. This is because the merger remnant is fully mixed at this stage. The abundances also change in between the collisions, as mass loss strips away the surface layers and reveals the deeper layers, but the change is not visible on the scale of the plots until the merger remnant becomes a Wolf-Rayet star. In sequences 1 and 2 the merger remnant undergoes a collision with a core heliumburning star at $3 \mathrm{Myr}$. This results in a strong increase in the carbon abundance (and oxygen, for sequence 2) and a decrease in the nitrogen abundance (through dilution). CNO cycling then converts the carbon into nitrogen, producing a nitrogen-rich WR

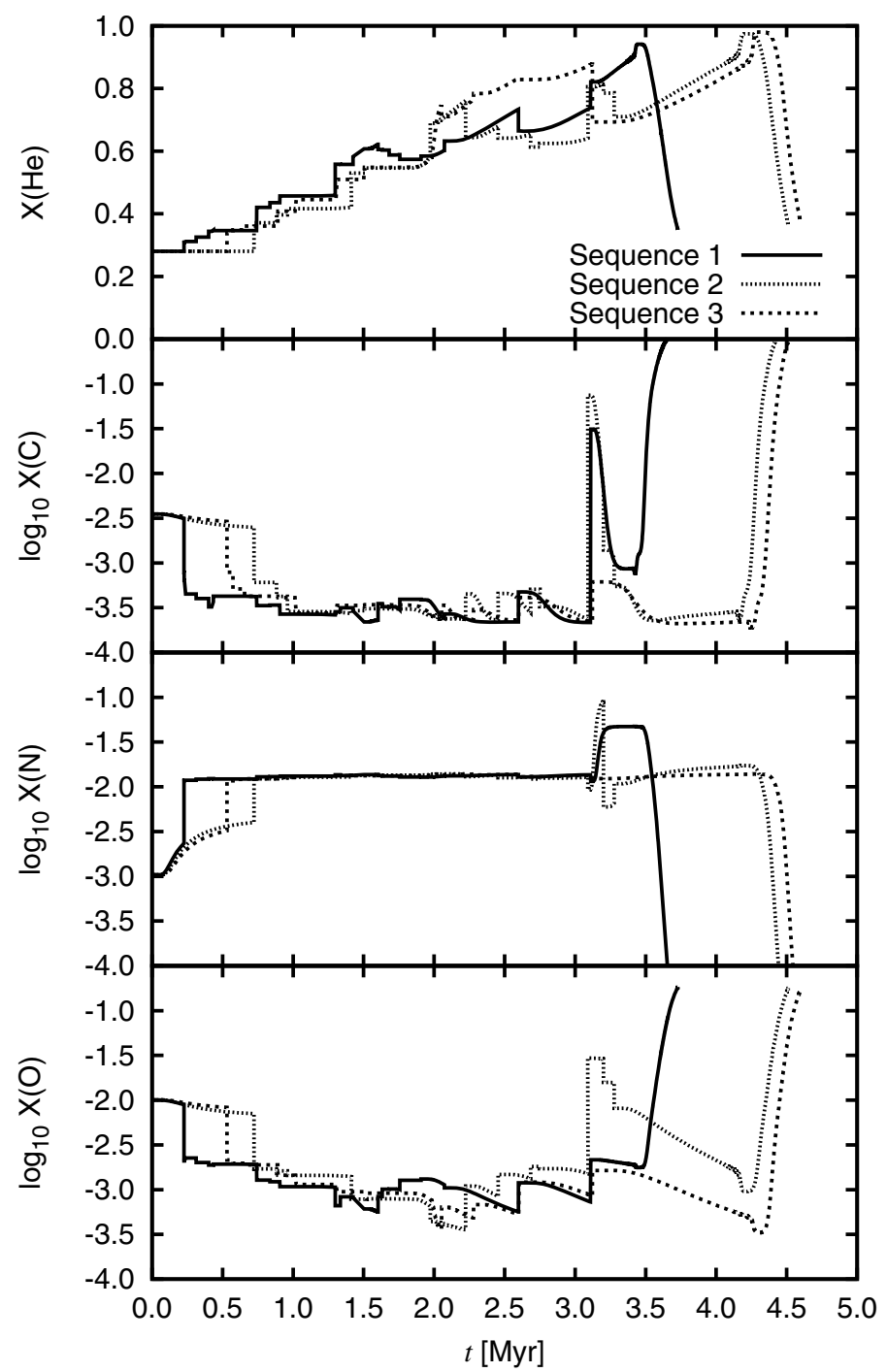

Fig. 5. Surface He, C, N and $\mathrm{O}$ abundances (by mass fraction) as a function of time (in Myr) for the three $Z=0.02$ sequences.

star. However, our assumption of complete mixing is unlikely to be valid for collisions with core helium-burning stars. More likely, most of the carbon and oxygen would remain in the core of the collision product and such a strong increase in surface $\mathrm{C}$ and $\mathrm{N}$ probably does not occur.

As the collision product continues to evolve after the end of the merger sequence, the surface helium abundance increases 


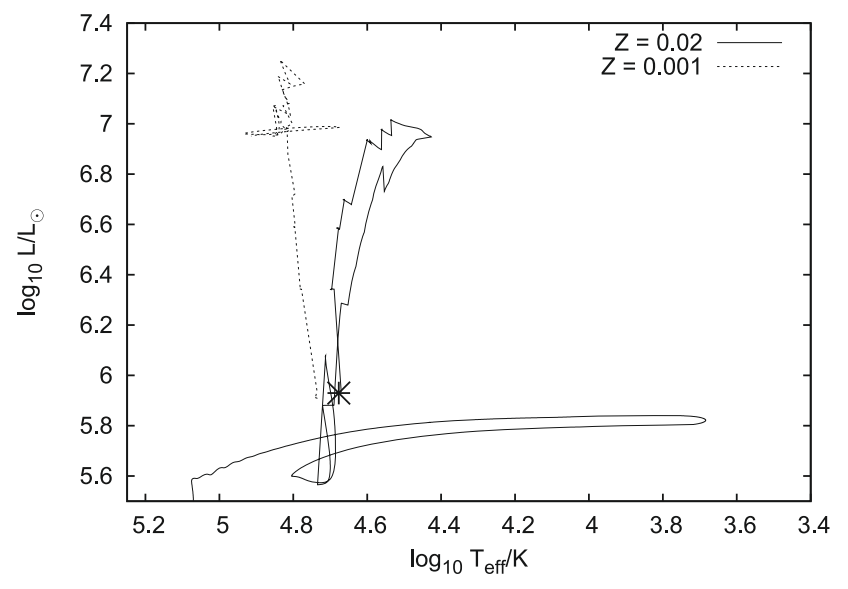

Fig. 6. Evolutionary track of the merger remnant of sequence 3 for $Z=$ 0.02 (solid line) and $Z=0.001$ (dashed line). Note that the $Z=0.001$ track remains much brighter than the $Z=0.02$ track.

until the hydrogen envelope has been lost and the surface is nearly pure helium. The stellar wind continues to expose deeper layers of the star, eventually revealing at the surface the ashes of helium burning. At this point, the surface nitrogen and helium abundance decrease while the carbon and oxygen abundances increase strongly. At the end of the evolution, carbon is the most abundant element on the surface.

The expulsion of gas from the cluster is usually attributed to supernova explosions, which are expected to start after about 3 Myr. The merger remnant loses most of its mass before this time. Table 6 gives the composition of the material lost from the merger remnant as well as the amount of material lost, split into three categories: ejecta resulting from the collisions, mass loss due to stellar wind during the merger sequence and mass loss due to stellar wind during the remaining evolution after the last collision. Most of the material is ejected in the form of a stellar wind between collisions, followed by the material that is lost during the collisions. The material that is lost by the collision product after the end of the merger sequence is significantly more helium-rich than the material that was lost before, which simply reflects the increased surface helium abundance of the collision product. The material lost from the collisions is less helium-rich than the material that is lost in the wind. This is partially due to the increase in the surface helium abundance between collisions (Fig. 5) and partially due to the fact that the estimated mass loss from the collision is larger for more equal masses and becomes smaller when the mass ratio is more extreme, which is the case for later collisions when the collision product is both massive and helium-rich.

In Col. 2 of Table 6 we give the total yield that would have been obtained from stellar winds if we had followed the evolution of the stars in the collision sequence individually. Because we only follow the evolution until the onset of carbon burning we only compare the pre-supernova yields. We first note that the single stars eject much less material than the merger sequence. This is because the merger sequence produces one $13.9 M_{\odot}$ black hole, while the single stars above $30 M_{\odot}$ all produce black holes of 8-24 $M_{\odot}$. The ejected material is also less helium-rich than the material that is lost from the merger remnant: the single star models lose $321 M_{\odot}$ of helium and $311 M_{\odot}$ of hydrogen $(\mathrm{He} / \mathrm{H} \approx 1)$, while the merger product loses $574 M_{\odot}$ of helium and $416 M_{\odot}$ of hydrogen $(\mathrm{He} / \mathrm{H} \approx 1.4)$. This is directly related to the large size of the convective core: for the merger remnant, $90 \%$ of the material has undergone nuclear processing in the core, which is a much larger fraction than for the population of single stars.

To compare the total yields from the merger sequence and the single stars we should, of course, also include the supernova yields. Here we can unfortunately only be qualitative because we do not have supernova yields for the merger remnant from our calculations and cannot rely on models from the literature. Supernova yields of normal massive single stars are of course available from the literature (e.g. Woosley \& Weaver 1995; Chieffi \& Limongi 2004; Hirschi et al. 2005).

Qualitatively, the net effect of the merger sequence is to suppress supernova nucleosynthesis: whereas the single stars produce multiple supernovae, the merger sequence produces only one supernova. In particular the merger sequence will produce less oxygen than a population of single stars.

\subsection{Metallicity effects}

Because mass-loss rates are lower at low metallicity we recalculated sequence 3 for $Z=0.001$. At this metallicity the mass-loss rates are about 13 times lower than for $Z=0.02$, which means that the remnant can become more massive.

The mass of the remnant after the last collision in the sequence is $263 M_{\odot}$. We followed the evolution of the merger product through core helium burning until the core helium abundance decreased to $Y_{\mathrm{c}} \approx 0.63$ (about one third of the core helium-burning lifetime), at which time the mass was $172 M_{\odot}$. Numerical problems prevented us from evolving this model further until carbon ignition. We extrapolated the mass loss as a linear function of the central helium abundance (rather than time) to the moment of helium exhaustion, which was found to give a good estimate of the final mass for $Z=0.02$. Based on this extrapolation we expect the final mass to be $\sim 120 M_{\odot}$ by the time of carbon ignition. The expected fate of this merger remnant is a pair-creation supernova (Langer et al. 2007; Portegies Zwart \& van den Heuvel 2007).

Because the opacity bump that gives rise to the core-halo structure is associated with iron, the core-halo structure does not appear at low metallicity and the collision product remains more compact. This is why in the bottom panel of Fig. 3 the radius of the $Z=0.001$ model (dash-dotted line) is smaller than the radius of the $Z=0.02$ model until $2 \mathrm{Myr}$, when the latter becomes a WR star. The radius is always smaller than the radius assumed in the $N$-body calculations.

The lower mass-loss rate makes it easier to build up more massive remnants, but the smaller radius reduces the probability of a collision and may prevent the occurrence of a runaway merger. The thermal timescale is also shorter at low metallicity, meaning that the star will reach thermal equilibrium faster after the collision. An additional complication is that the lifetime of the stars is reduced at lower metallicity (because of their higher mass) so that there is less time to form the merger sequence. Fully self-consistent dynamical models (in which the stellar evolution, stellar collisions and stellar dynamics are all treated consistently) are necessary to determine which of these effects dominates in practice.

\section{Discussion and conclusions}

\subsection{Mass loss and rotation}

The main uncertainty in our models is the adopted mass-loss rate. We have used established mass-loss prescriptions from the literature. Our adopted Vink et al. (2001) rate is derived for stars 
that are further away from their Eddington luminosity than our collision products and is likely to be an underestimate of the true mass-loss rate for our stars.

We apply WR mass loss rates when the surface hydrogen abundance $X_{\mathrm{s}}<0.4$. This transition is somewhat arbitrary and ideally we would use a single mass loss recipe that predicts the mass-loss rate as a function of the local luminosity, temperature, effective gravity and composition. No such recipe is available in the literature at the time of writing.

The mass-loss rate can become very high during the WR phase due to our extrapolation of the empirical rate to higher luminosity than for which it was derived. We made sure that the power used to drive the wind is always less than that provided by the star's luminosity (Owocki et al. 2004). When the collision products first become WR stars, the mass-loss rate is still very high. In part this is due to the sudden transition to WR mass loss rates when $X_{\mathrm{s}}$ drops below 0.4 . We expect that if the transition is made more smoothly the mass-loss rate would increase earlier and avoid the high peak value found in our current models. This again stresses the need for a unified single mass loss prescription.

Our collision products have luminosities close to their Eddington luminosity and for a substantial amount of time exceed the Humphreys-Davidson limit. Stars close to this limit become luminous blue variables which can lose a large amount of mass in outbursts. A model that describes mass loss from stars that exceed their Eddington luminosity is the so-called porosity model (Owocki et al. 2004; Owocki \& van Marle 2008). Our models come close, but do not exceed their Eddington luminosity. We made one trial run with the mass-loss rate artificially enhanced by a factor of 50 and found that although the star acquires new material from collisions this is not enough to overcome the high mass loss rate and the star is very quickly stripped of its mass.

We have ignored rotation in this work. Rotational mixing is expected to be unimportant because our models are almost fully convective. Rapid rotation is also expected to enhance the mass-loss rate from stars, especially close to the Eddington limit (Maeder \& Meynet 2000a). Off-axis collisions almost always produce remnants that need to lose angular momentum before they can reach thermal equilibrium (Sills et al. 2005). How this angular momentum is lost and how much mass is lost in the process is currently poorly understood, further increasing the uncertainty in the mass loss rate.

\subsection{Implications for nucleosynthesis}

Anomalous chemical abundances in globular clusters are the topic of much debate in the literature. Some stars show $\mathrm{N}$ and $\mathrm{Na}$ enhancement but $\mathrm{O}$ depletion and some show indications of He enhancement. Current scenarios that attempt to explain these chemical anomalies require pollution of the interstellar medium by massive stars (Decressin et al. 2007) or AGB stars (e.g. D'Ercole et al. 2008) in the central regions of the cluster and a second episode of star formation, followed by the loss of most of the first generation of low mass stars. In this context it is interesting to consider the abundance pattern that would result from a collisional runaway, not just to compare it with other scenarios but also because this can give us a chemical signature that would allow us to determine whether or not a runaway occurred in the past in a cluster observed today.

The simplified nuclear network in our evolution code only follows the main isotopes of the elements listed in Table 6. In particular, we do not follow the evolution of isotopes of $\mathrm{Na}, \mathrm{Mg}$ or Al. However, we can make a few qualitative estimates. The material that is lost from the collision remnant is helium-rich and shows the signature of $\mathrm{CNO}$ processing ( $\mathrm{N}$ enhancement, $\mathrm{C}$ depletion). The material is also ejected in the centre of the cluster. In this sense, the expected abundance pattern would be similar to that generated by the other scenarios mentioned above. We do expect the collision sequence to produce less oxygen and metals than a population of single stars because in this case there is only one star that can generate a supernova and that will not eject a large amount of material, given that its pre-supernova mass is very small. Nevertheless it is difficult to identify how it could be distinguished from the other scenarios observationally without more detailed nucleosynthesis. It is possible to infer yields for a wider range of isotopes from our models using a nucleosynthesis post-processing code. We plan to present the results of such a study, including an estimate for the supernova yields in a follow-up paper.

Although the collisional runaway causes more material to be returned to the interstellar medium than the equivalent population of single stars would have done, the amount of ejected material is still small: observed helium-rich populations generally seem to comprise about $15-20 \%$ of the stars in the cluster (Pumo et al. 2008), which is larger than the fraction of mass lost from the merger remnant compared to the total cluster mass. Smilar to other scenarios, this scenario requires that many first generation stars are lost from the cluster. It appears, however, that this may be feasible (D'Ercole et al. 2008). Multiple collisional runaways, as found by Gürkan et al. (2006), can of course increase our estimate of the yields.

\subsection{Conclusions and outlook}

We find that the end result of a runaway merger at solar metallicity is a $\sim 100 M_{\odot}$ WR star after the final collision that produces a $\sim 10 M_{\odot}$ black hole. Most of the mass is lost in the form of a stellar wind enriched in $\mathrm{N}$ and He. At lower metallicity the mass-loss rates are reduced and the remnant mass can be higher ( $\left.260 M_{\odot}\right)$, possibly leading to a pair-creation supernova. In all cases strong mass loss, in particular during the WR phase, prevents the formation of an intermediate-mass black hole. Because we have decoupled the $N$-body dynamics from the stellar evolution in this work the mass and radius of our detailed evolution models are different from those used in the $N$-body run, which would change the collision probability. We do not expect this to alter our conclusions because the physical mechanism that prevents the formation of the intermediate-mass black hole, the strong WR wind, does not depend on the dynamics.

Nevertheless it is important to perform fully self-consistent simulations of collisional runaways. Calculations like those by Belkus et al. (2007) in which the stellar evolution is treated with an analytic recipe, are an important step in this direction. We will extend this in future work by coupling our stellar evolution code to a dynamics code in the MUSE framework (Portegies Zwart et al. 2008). Calculations at lower $Z$ are especially interesting since remnant masses can be higher due to a reduced stellar wind, but on the other hand collisions are less likely because the stars are more compact.

Acknowledgements. Evert thanks Jorick Vink and Allard Jan van Marle for useful discussion about mass-loss rates from luminous stars in general and Henny Lamers for the Wolf-Rayet mass-loss rates in particular. He also thanks Rob Izzard for more general discussion. We thank Georges Meynet for providing many useful and inspiring comments that greatly improved this paper. E.G. and E.G. are supported by NWO under grants 614.000.303 and 635.000.303. S.d.M. 
is partially supported by NOVA. The research was conducted in the context of the MODEST collaboration.

\section{References}

Anders, E., \& Grevesse, N. 1989, Geochim. Cosmochim. Acta, 53, 197 Belczynski, K., Kalogera, V., \& Bulik, T. 2002, ApJ, 572, 407

Belkus, H., Van Bever, J., \& Vanbeveren, D. 2007, ApJ, 659, 1576

Bettwieser, E., \& Sugimoto, D. 1984, MNRAS, 208, 493

Böhm-Vitense, E. 1958, ZsAp, 46, 108

Chieffi, A., \& Limongi, M. 2004, ApJ, 608, 405

de Jager, C., Nieuwenhuijzen, H., \& van der Hucht, K. A. 1988, A\&AS, 72, 259

Decressin, T., Charbonnel, C., \& Meynet, G. 2007, A\&A, 475, 859

D’Ercole, A., Vesperini, E., D'Antona, F., McMillan, S. L. W., \& Recchi, S. 2008, ArXiv e-prints

Eggleton, P. P. 1971, MNRAS, 151, 351

Eggleton, P. P. 1972, MNRAS, 156, 361

Eggleton, P. P., Fitchett, M. J., \& Tout, C. A. 1989, ApJ, 347, 998

Eldridge, J. J., \& Tout, C. A. 2004, MNRAS, 348, 201

Eldridge, J. J., \& Vink, J. S. 2006, A\&A, 452, 295

Elmegreen, B. G. 1999, ApJ, 515, 323

Endal, A. S., \& Sofia, S. 1976, ApJ, 210, 184

Ferguson, J. W., Alexander, D. R., Allard, F., et al. 2005, ApJ, 623, 585

Figer, D. F. 2005, Nature, 434, 192

Figer, D. F., Najarro, F., Morris, M., et al. 1998, ApJ, 506, 384

Freitag, M., Gürkan, M. A., \& Rasio, F. A. 2006, MNRAS, 368, 141

Gaburov, E., Glebbeek, E., Portegies Zwart, S., \& Pols, O. 2008a, in preparation

Gaburov, E., Lombardi, J. C., \& Portegies Zwart, S. 2008b, MNRAS, 383, L5

Glebbeek, E., Pols, O. R., \& Hurley, J. R. 2008, A\&A, 488, 1007

Gürkan, M. A., Fregeau, J. M., \& Rasio, F. A. 2006, ApJ, 640, L39

Heger, A., Langer, N., \& Woosley, S. E. 2000, ApJ, 528, 368

Hillier, D. J., Davidson, K., Ishibashi, K., \& Gull, T. 2001, ApJ, 553, 837

Hirschi, R., Meynet, G., \& Maeder, A. 2005, A\&A, 433, 1013

Humphreys, R. M., \& Davidson, K. 1979, ApJ, 232, 409

Iglesias, C. A., \& Rogers, F. J. 1996, ApJ, 464, 943

Ishii, M., Ueno, M., \& Kato, M. 1999, PASJ, 51, 417

Kippenhahn, R., \& Weigert, A. 1990, Stellar Structure and Evolution, XVI (Berlin, Heidelberg, New York: Springer-Verlag), also Astronomy and Astrophysics Library, 468

Kormendy, J., \& Richstone, D. 1995, ARA\&A, 33, 581
Kudritzki, R. P. 2002, ApJ, 577, 389

Langer, N., Norman, C. A., de Koter, A., et al. 2007, A\&A, 475, L19

Leonard, P. J. T., \& Livio, M. 1995, ApJ, 447, L121

Lombardi, Jr., J. C., Rasio, F. A., \& Shapiro, S. L. 1996, ApJ, 468, 797

Lombardi, Jr., J. C., Warren, J. S., Rasio, F. A., Sills, A., \& Warren, A. R. 2002, ApJ, 568, 939

Maeder, A., \& Meynet, G. 2000a, A\&A, 361, 159

Maeder, A., \& Meynet, G. 2000b, ARA\&A, 38, 143

Makino, J. 2001, in Dynamics of Star Clusters and the Milky Way, ed. S. Deiters, B. Fuchs, A. Just, R. Spurzem, \& R. Wielen, ASP Conf. Ser., 228, 87 McCrady, N., Gilbert, A. M., \& Graham, J. R. 2003, ApJ, 596, 240

Nugis, T., \& Lamers, H. J. G. L. M. 2000, A\&A, 360, 227

Owocki, S. P., \& van Marle, A. J. 2008, ArXiv e-prints, 801

Owocki, S. P., Gayley, K. G., \& Shaviv, N. J. 2004, ApJ, 616, 525

Petrovic, J., Pols, O., \& Langer, N. 2006, A\&A, 450, 219

Pinsonneault, M. H., Kawaler, S. D., Sofia, S., \& Demarque, P. 1989, ApJ, 338, 424

Pols, O. R., Tout, C. A., Eggleton, P. P., \& Han, Z. 1995, MNRAS, 274, 964

Portegies Zwart, S. F., \& McMillan, S. L. W. 2002, ApJ, 576, 899

Portegies Zwart, S. F., Makino, J., McMillan, S. L. W., \& Hut, P. 1999, A\&A, 348, 117

Portegies Zwart, S. F., McMillan, S. L. W., Hut, P., \& Makino, J. 2001, MNRAS, 321,199

Portegies Zwart, S. F., Baumgardt, H., Hut, P., Makino, J., \& McMillan, S. L. W. 2004, Nature, 428, 724

Portegies Zwart, S. F., \& van den Heuvel, E. P. J. 2007, Nature, 450, 388

Portegies Zwart, S., McMillan, S., Harfst, S., et al. 2008, New Astron., accepted Pumo, M. L., D’Antona, F., \& Ventura, P. 2008, ApJ, 672, L25

Rogers, F. J., \& Iglesias, C. A. 1992, ApJS, 79, 507

Seaton, M. J., Yan, Y., Mihalas, D., \& Pradhan, A. K. 1994, MNRAS, 266, 805

Sills, A., Lombardi, Jr., J. C., Bailyn, C. D., et al. 1997, ApJ, 487, 290

Sills, A., Adams, T., \& Davies, M. B. 2005, MNRAS, 358, 716

Suzuki, T. K., Nakasato, N., Baumgardt, H., et al. 2007, ApJ, 668, 435

Vink, J. S., \& de Koter, A. 2005, A\&A, 442, 587

Vink, J. S., de Koter, A., \& Lamers, H. J. G. L. M. 2000, A\&A, 362, 295

Vink, J. S., de Koter, A., \& Lamers, H. J. G. L. M. 2001, A\&A, 369, 574

Weidner, C., \& Kroupa, P. 2004, MNRAS, 348, 187

Woosley, S. E., \& Weaver, T. A. 1995, ApJS, 101, 181

Yoon, S.-C., \& Langer, N. 2005, A\&A, 443, 643

Yungelson, L. R., van den Heuvel, E. P. J., Vink, J. S., Portegies Zwart, S. F., \& de Koter, A. 2008, A\&A, 477, 223 